\title{
From Tele-remote Operation to Semi-automated Wheel-loader
}

\author{
Siddharth Dadhich, Ulf Bodin, Fredrik Sandin and Ulf Andersson \\ Department of Computer Science, Electrical and Space Engineering \\ Luleå University of Technology, Luleå, Sweden \\ Email: \{siddharth.dadhich, ulf.bodin, fredrik.sandin, ulf.andersson\}@ltu.se
}

\begin{abstract}
This paper presents experimental results with tele-remote operation of a wheel-loader and proposes a way towards semi-automation. The different components of the tele-remote setup are described in the paper. Short loading cycle is an operation cycle, commonly used at quarry and construction sites for moving gravel from piles onto trucks. We present results from short loading cycle experiments with three operators comparing productivity between teleremote operation and manual operation. Productivity loss of $42 \%$ with tele-remote operation motivates the case for more automation. We propose a method to automate the bucketfilling step, which is one of the key functionality of a wheelloader.
\end{abstract}

Index Terms - wheel-loader; tele-operation; construction; quarry; automation; bucket-filling

\section{INTRODUCTION}

Tele-operation of commercial vehicles is interesting for both safety and productivity reasons. The on-board operation of such machines can be unsafe in certain applications. For example, in underground mining there are situations when loading of blasted material is postponed awaiting ventilation to clean the air for workers to enter the mine [1]. At mines, construction and quarry sites, operators are required to operate the equipment for long hours in stretch especially when the working-site is far from their office space. Exposure to noise, vibration and ergonomics issues for long durations produces stress and strain on their body.

A wheel-loader (shown in Fig. 1) is a versatile machine used to move materials in many different industries. Full automation of many tasks that are needed to be performed by wheel-loaders is difficult [2] and the bucket-filling task is, in particular, hard to automate. The skilled drivers use many of their human senses to achieve high productivity at low fuel consumption without causing unnecessary wear and tear on the machine. Tele-remote operation can improve both safety and productivity by providing comfortable work environment and eliminating the time needed to commute to the working-sites.

Short loading cycle is a common operation cycle at construction and quarry sites. In this cycle, the machine repeatedly loads the material from a pile and then dumps it onto a dumper truck. An illustration of a typical short loading cycle is shown in Fig. 2.

Our tele-remote setup uses $2.4 \mathrm{GHz}$ IEEE 802.11n radio to transmit the video and other data including control signals. The control station that we use is similar to a machine's cab further modified with display screens to view $360^{\circ}$ live video streams.

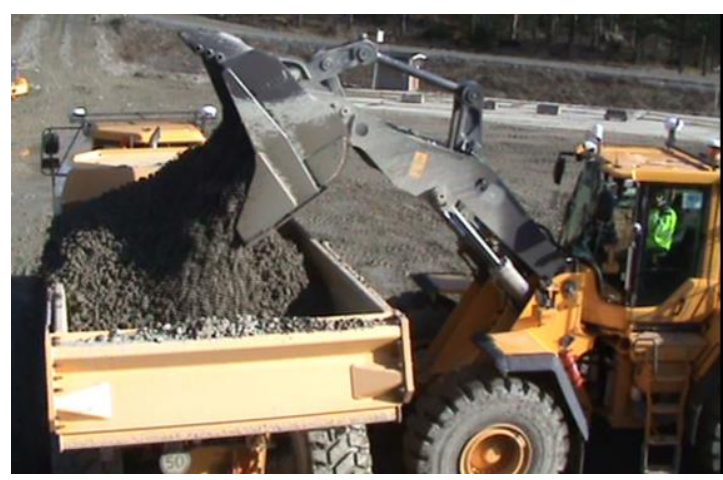

Figure 1. Our experimental wheel-loader.

Tele-remote operation is a step toward full automation of construction machines. Due to the difficulties in modeling the bucket-pile interactions, automation of the bucket-filling task for loaders and excavator has been an open problem since three decades [3].

Tele-operation of short-cycle loading is known to be less efficient compared to manual operation [4]. In this paper, we quantify this gap with our remote control setup highlighting the difficulties in tele-remote operation. We also propose and evaluate a method to automate the key functionality of a wheel-loader i.e. the bucket-filling.

\section{RELATED WORK}

Tele-remote operation and automation of commercial vehicles is under development in industry [5]. In academia, the main work is towards improving the perception, for example, by providing visualizations of different perspectives of the construction machine in its environment by cameras mounted on the machine itself [6]. In our setup, we use an isometric perspective of the machine itself, excluding the environment. The dome display environment, investigated in [7], is shown to improve the perception compared to a flat screen display setup, which we have used.

Reference [8], similar to our work, present results from remote-operation of an excavator over a wireless IP network. They use a head mount display (HMD) with end to end latency of 180 milliseconds and report $164 \%$ 
increase in cycle time of operation for remote control, compared to manual operation.

Related research on automatic bucket-filling is the work done on Load-haul-dump (LHD) machines, which are a variation of wheel-loaders adapted for underground mines. For example, [9] developed a compliance controller for the velocity of the tilt cylinder that uses the hydraulic pressures in the lift and tilt pistons as inputs. Reference [10] shows that, the method proposed in [9] works in practice and also performs better than human drivers on LHD machines. However, in our work, we propose a more generic solution to the bucket-filling problem, applicable to different types of machines and environment conditions.

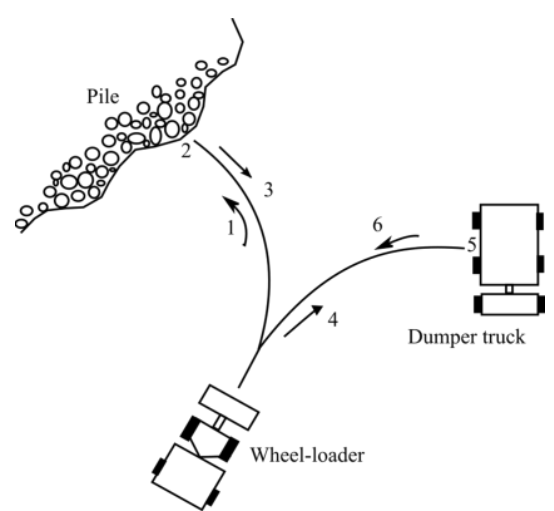

Figure 2. Short loading cycle. The steps performed by a wheel loader in one operation cycle are: 1: Approach to the pile, 2: Bucket-filling, 3: Reversing from the pile 4: Approach the dumper, 5: Dumping, 6: Reversing from the dumper.

\section{EXPERIMENT SETUP}

\section{A. Wheel-loader}

The experiment consists of a Volvo L180 wheel loader, which is instrumented to enable the functionalities needed for remote-operation. To control the machine externally, we use an industrial PC which has slots for Canbus cards which are used to control the machine by sending signals to the machine ECUs (Engine control units). The software in one of the ECUs is modified to receive control signals for lift, tilt, gas, brake, gear and steering. Furthermore, we have six IP cameras, a microphone for audio; a GPS unit for localization, an inertial-measurement-unit (IMU) mounted on the chair in the machine and a WiFi access point (AP) for communication.

\section{B. Communication}

In Fig. 3, the test setup for the remote control experiment is shown. The remote control station and the wheel-loader are connected by a part-wired (Ethernet and fiber) and part-wireless (IEEE802.11n) network. The wheel-loader and the control station are in two different locations $\sim 800$ meters apart. Most of the devices shown in Fig. 3 have an Ethernet interface except the IMU and GPS which are connected to industrial PC via Canbus and Serial-bus respectively. We used IEEE $802.11 \mathrm{n}$ as the radio link for this setup because it is widely deployed in industries such as mining and construction where wheel loaders are commonly used.
In total, we have four types of data streams over the network as shown in Fig. 4. The data streams from cameras and microphone are seven individual streams representing most of the traffic ( $99 \%$ when the machine is in motion).

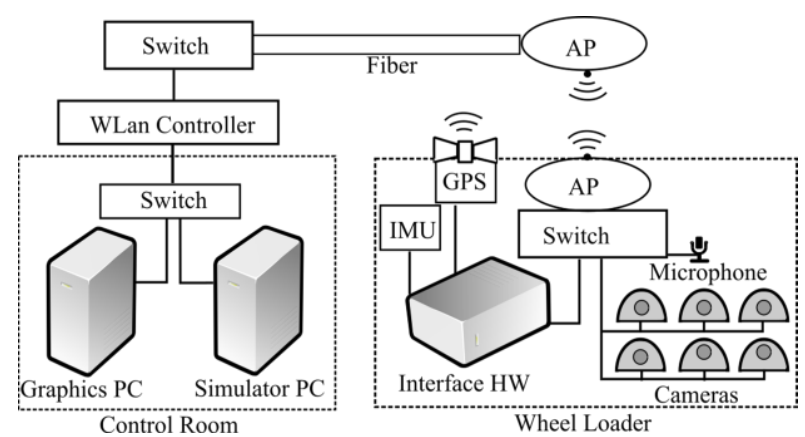

Figure 3. The remote-control setup.

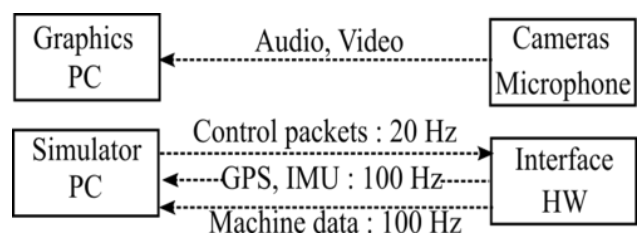

Figure 4. Data streams between the machine and the control station.

\section{Remote Control Station}

The remote control station (Fig. 5) is a motion platform with similar controls panels as a machine. This platform has roll and pitch movements and is commonly used as a simulator to train new operators of wheel-loaders and excavators. The platform has been modified with screens and additional software, to send the stream of control data. An HMI program is developed which receives the feedback streams from cameras, GPS and machine data and displays different views (top, side and isometric) of the machine and updates a simulated dashboard. The IMU data stream is used to move the motion platform.

\section{Video Setup}

We used six cameras to transmit live video from around the machine. These cameras support both MJPEG and H.264 video protocols and can transmit full HD (1980x1080) resolution. However, we operated them at $1280 \times 720$ with H.264 due to bandwidth limitation of the Wi-Fi network for real-time live video with mobile clients. Furthermore, we limit the bandwidth used by each camera using H.264 parameters.

The latency in the video transmission depends on 1) the camera's hardware and firmware (image processing and encoding) 2) the network and 3) the receiving client (decoding and display). The latency of the camera we use (Panasonic WV-SBV131M) is found out to be in the range of 150-250 milli-seconds depending on H.264 parameters, which is in line with other IP cameras as discussed in [11]. 


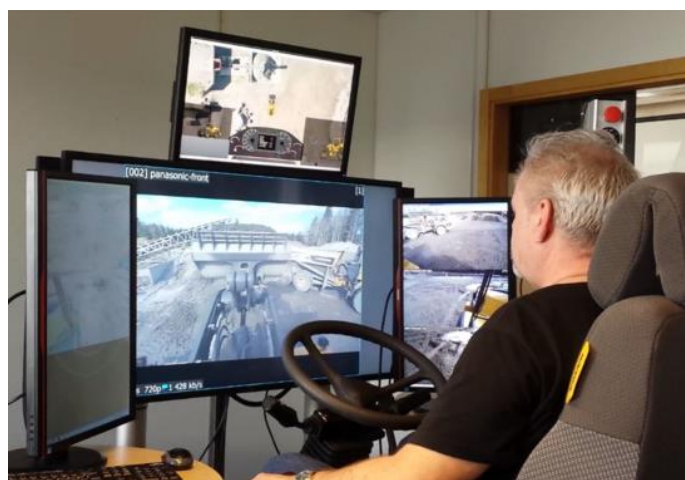

Figure 5. The Remote control station.

\section{E. Procedure}

The operators were asked to fill a Volvo A25 truck with three full buckets in a short loading cycle. The material used in this experiment was medium-course gravel (8-32 $\mathrm{mm}$ in diameter).

The operators were trained for two hours on the remote control station and before the experiment started, operators were given some time to fill a few trucks to warm-up before they were measured and recorded. The three operators we used in this study have a lot of experience with manual operation of wheel-loaders. During experiment, each operator filled the truck three times (nine short loading cycle in total) in manual operation and then three times remotely.

We logged relevant machine signals; weight of the dumper, networking data, and recorded videos of the machine in both manual and remote operation to measure the differences.

\section{RESULTS AND DISCUSSION}

In this experiment, we consider productivity, in terms of the amount of material moved per unit time (tons/hour), as the performance parameter. Productivity for a wheel loader is a function of payload and cycle time. It decreases due to less filled buckets and long operation cycle times.

We observed that cycle time of operation for remote operation increased by $70 \%$ from manual operation, which explains the $42 \%$ loss in productivity we observed for the remote-operation. Fig. 6 shows that the average cycle time of operation increases for all parts of short loading cycle. The maximum increase is for steps four and five of the short loading cycle, in which the machine approaches and dumps the material into the truck.

We also observed that the average bucket weight for remote operation was lower compared to manual operation. As shown in Table I, the difference in average bucket weight was however very small and on average, the operators managed to fill the bucket $(97 \%$ of how much they filled in manual).

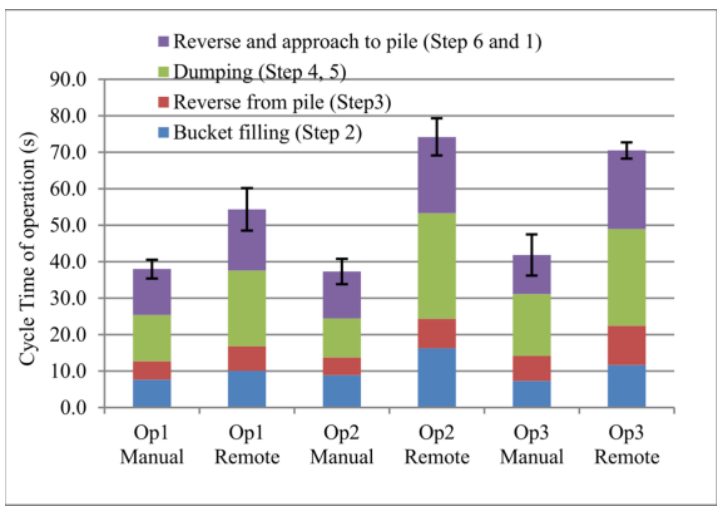

Figure 6. Average cycle time for the three operators (Op) in manual and remote operation where the standard deviation is shown for the complete cycles.

TABLE I. AVERAGE BUCKET WEIGHTS (TON) FOR THE THREE OPERATORS (OP)

\begin{tabular}{|l|c|c|c|}
\hline & Op 1 & Op 2 & Op 3 \\
\hline Manual & 7.62 & 7.94 & 6.99 \\
\hline Remote & 7.35 & 7.72 & 6.81 \\
\hline
\end{tabular}

The results show that drivers were slower during remote-operation. The increase in cycle time of operation on remote has possibly several reasons. The lack of perception of being in the machine is likely the main issue. For example, the operators in manual operation use their vestibular system (located in the ear) to detect linear (gravito-inertial forces) and rotational accelerations in 3D [12]. The motion platform used in the experiment provides only the roll and pitch movements and hence the operators fail to judge the linear speed/acceleration during driving.

Lack of depth perception, which arises from human vision being replaced by $2 d$ camera-feed, presents a drawback of the remote-operation. Additionally, some network issues, that arises when all video streams are running, results in small but noticeable glitches in the one of the cameras at a time with a frequency of around $1 \mathrm{~Hz}$.

The difficulties with the remote-operation motivate the case for more automation. A major roadblock to full automation of wheel-loaders is the bucket-filling step. In the next section, we present our on-going work on automating bucket-filling for a wheel-loader.

\section{AUTOMATIC BUCKET-FILLING}

A fully autonomous loading operation is known to be difficult and has been an open area of research for a long time. Although tele-operated LHD machines have been used in underground mines for more than ten years [3], a fully automated system of an LHD machine has never been demonstrated.

To address this, [4] developed a bucket-filling algorithm for tele-remote operated LHD machines to load blasted rock in an underground mine. The resulting semiautomated operation was less productive than manual operation and tele-operation was therefore discontinued.

Reference [13] proposes a machine learning based function for bucket-filling to load medium-course gravel. We build upon this work and see if machine learning 
models can predict the motion of a wheel loader's pistons in terms of the velocity of lift/tilt cylinders.

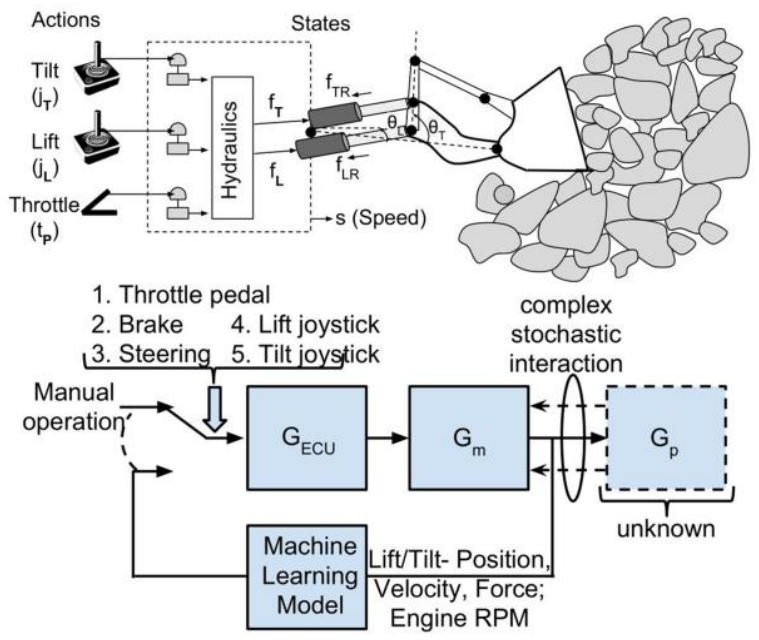

Figure 7. Block diagram of the loading process (bottom) and variables (top) for the machine $(\mathrm{Gm})$ and the pile $\left(\mathrm{G}_{\mathrm{p}}\right)$. The operator actions include lift and tilt joystick commands and movement of the throttle pedal.

\section{A. Data}

The data used to develop the following models have been provided by our industrial partners. The data contains variables such as the angles of lift and tilt joints and hydraulic pressures in the lift and tilt cylinders of a Volvo L120G wheel loader that is used by an expert driver to load medium-course gravel $(8-32 \mathrm{~mm}$ in diameter). Fig. 7 illustrates the automatic loading problem in terms of a control block diagram and the interaction forces between the machine and pile. The hydraulic system in the convectional diesel engine powered machines is a multi-variable system where the forces applied to lift and tilt pistons come from a non-linear coupled function of operator actions as shown in (1).

$$
f_{L}, f_{T}=f\left(j_{L}, j_{T}, t_{P}\right)
$$

The resistance forces from the pile $\left(f_{L R}, f_{T R}\right)$ cannot be measured or accurately modeled, and this poses the main challenge in the bucket-filling problem. The lift and tilt angles $\left(\theta_{L}, \theta_{T}\right)$ define the trajectory of the bucket through the pile.

\section{B. Cascade Model}

First a linear regression model was tested which failed to capture the correct dynamics of the driver's use of the lift and tilt actions. We conclude that the actions of drivers cannot be predicted by a linear model and non-linear methods are needed. In reality, the lift and tilt actions of the driver are discrete and related to the dynamics of the pile, the bucket-filling process, and the sensory ques and experience (priors) of the driver.

We extend the linear regression model with a classification model in an attempt to approximate the driver's decision to use the lift and tilt actions. This is motivated from the observation that expert drivers use lift and tilt cylinders alternatively.

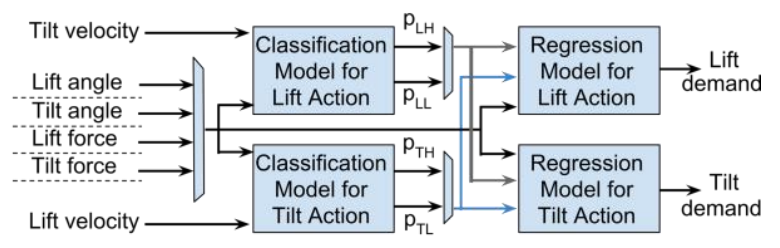

Figure 8. Machine learning model for predicting the motion of wheel-loader pistons during bucket-filling.

Fig. 8 illustrates the proposed scheme of cascaded models for estimation of lift/tilt velocity demands. First, the classification model predicts (based on data presented during training) the decision probability of high $\left(p_{L H}, p_{T H}\right)$ and low $\left(p_{L L}, p_{T L}\right)$ values of the lift and tilt actions. The classification blocks in Fig. 8 contain two classification models each, one for high and one for low action. The structure of the data used for the training of classification and regression models is shown in Table II. The threshold parameters $\dot{\theta}_{L H t h}, \dot{\theta}_{L L t h}, \dot{\theta}_{T H t h}, \dot{\theta}_{T L t h}$ for training the classifiers have been handcrafted.

TABLE II. STRUCTURE OF THE TRAINING DATA FOR THE CASCADE MODEL

\begin{tabular}{|l|c|c|}
\hline \multicolumn{1}{|c|}{$\begin{array}{c}\text { Model } \\
\text { type }\end{array}$} & Feature vector & Output \\
\hline $\mathrm{C}^{\mathrm{a}}$ for $\mathrm{p}_{\mathrm{LH}}$ & {$\left[\dot{\theta_{\mathrm{T}}}, \theta_{\mathrm{L}}, \theta_{\mathrm{T}}, \mathrm{f}_{\mathrm{L}}, \mathrm{f}_{\mathrm{T}}\right]$} & $\dot{\theta_{\mathrm{L}}}>\dot{\theta}_{\mathrm{LHth}}$ \\
\hline $\mathrm{C}$ for $\mathrm{p}_{\mathrm{LL}}$ & {$\left[\dot{\theta_{\mathrm{T}}}, \theta_{\mathrm{L}}, \theta_{\mathrm{T}}, \mathrm{f}_{\mathrm{L}}, \mathrm{f}_{\mathrm{T}}\right]$} & $\dot{\theta_{\mathrm{L}}}<\dot{\theta}_{\mathrm{LLth}}$ \\
\hline $\mathrm{C}$ for $\mathrm{p}_{\mathrm{TH}}$ & {$\left[\dot{\theta_{\mathrm{L}}}, \theta_{\mathrm{L}}, \theta_{\mathrm{T}}, \mathrm{f}_{\mathrm{L}}, \mathrm{f}_{\mathrm{T}}\right]$} & $\dot{\dot{\theta}_{\mathrm{T}}}>\dot{\theta}_{\mathrm{THth}}$ \\
\hline $\mathrm{C}$ for $\mathrm{p}_{\mathrm{TL}}$ & {$\left[\dot{\theta_{\mathrm{L}}}, \theta_{\mathrm{L}}, \theta_{\mathrm{T}}, \mathrm{f}_{\mathrm{L}}, \mathrm{f}_{\mathrm{T}}\right]$} & $\dot{\theta_{\mathrm{T}}}<\dot{\theta}_{\mathrm{TLth}}$ \\
\hline $\mathrm{R}^{\mathrm{b}}$ for $\mathrm{j}_{\mathrm{L}}$ & {$\left[\mathrm{p}_{\mathrm{LH}}, \mathrm{p}_{\mathrm{LL}}, \mathrm{p}_{\mathrm{TH}}, \mathrm{p}_{\mathrm{TL}}, \theta_{\mathrm{L}}, \theta_{\mathrm{T}}, \mathrm{f}_{\mathrm{L}}, \mathrm{f}_{\mathrm{T}}\right]$} & $\dot{\theta_{\mathrm{L}}}$ \\
\hline $\mathrm{R}$ for $\mathrm{j}_{\mathrm{T}}$ & {$\left[\mathrm{p}_{\mathrm{LH}}, \mathrm{p}_{\mathrm{LL}}, \mathrm{p}_{\mathrm{TH}}, \mathrm{p}_{\mathrm{TL}}, \theta_{\mathrm{L}}, \theta_{\mathrm{T}}, \mathrm{f}_{\mathrm{L}}, \mathrm{f}_{\mathrm{T}}\right]$} & $\dot{\theta_{\mathrm{T}}}$ \\
\hline
\end{tabular}

Instead of using binary output, we use the confidence values $\left(p_{L H}, p_{L L}, p_{T H}, p_{T L}\right)$ from each classifier as inputs to the regression model which predicts the velocity demands for lift and tilt pistons.

\section{Simulation Result}

The performance of a machine learning model is assessed on the basis of its generalization capabilities on unseen data. In Fig. 9, we show a test example of how the proposed cascaded network of machine learning models performs on an unseen bucket-filling example. The model approximates the velocity of lift and tilt cylinders quite well and it can be observed that the prediction captures the on-off nature of the lift and tilt commands.

\section{CONCLUSION AND FUTURE WORK}

Tele-remote operation of short loading cycle with wheel-loaders is challenging. The constrained human perception when using tele-remote setup makes it difficult to operate efficiently. We found that the remote operation with this setup results in productivity loss of $42 \%$ which is mainly due to the increase in the time of operation.

The depth perception for the remote-operation needs to be improved by integrating technologies like ultra-sonic 
sensor and lidar into the current HMI. A reliable wireless medium which is less sensitive to varying radio conditions is also essential for the remote operation. The requirement from such a radio should be low jitter along with tolerable latency.
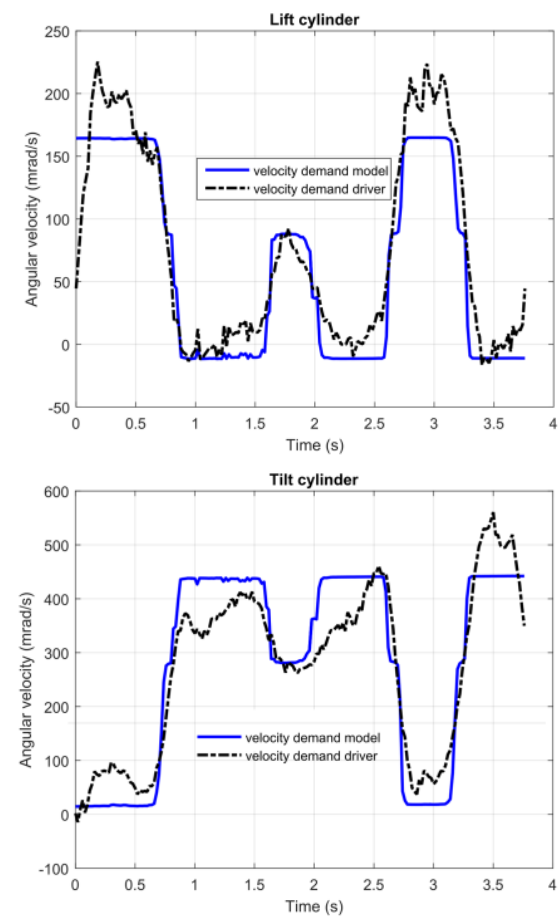

Figure 9. Prediction of lift/tilt velocity demand from the proposed model.

We propose an automatic bucket-filling function based on machine learning using data from manual operation. Classification and regression models are used in cascade to build a system that approximates the lift and tilt actions from the velocities of lift/tilt pistons. The classification models were able to predict driver behavior in terms of the decision to use the lift and tilt actions.

The first implementation of the machine learning based bucket-filling function gives promising results. In future, we plan to conduct more field experiments to validate the proposed bucket-filling algorithm. Reinforcement learning methods will be investigated to further improve the efficiency of the bucket-filling function.

\section{ACKNOWLEDGMENT}

The work presented in this article is supported by the Tempe foundation under the contract 2014-01882. We thank Erik, Torbjörn, Benny, Ola and Michael who contributed in the experiments. We acknowledge the support of our industrial partners, Volvo CE and Oryx Prototyping.

\section{REFERENCES}

[1] S. Dadhich, U. Bodin, and U. Andersson, "Key challenges in automation of earth-moving machines," Automation in Construction, vol. 68, pp. $212-222,2016$, doi:10.1016/j.autcon.2016.05.009.

[2] A. Hemami and F. Hassani, "An overview of autonomous loading of bulk material," in Proc. 26th International Symposium on Automation and Robotics in Construction and Mining, 2009, pp. 405-411, doi:10.22260/ISARC2009/0013.

[3] G. J. Maeda, "Learning and Reacting with Inaccurate Prediction: Applications to Autonomous Excavation," Ph.D. dissertation, School of Aerospace, Mechanical and Mechatronic Engineering, The University of Sydney, 2013.

[4] U. Andersson, "Automation and Traction Control of Articulated Vehicles," Ph.D. dissertation, Department of Computer Science, Electrical and Space Engineering, Luleå University of Technology, 2013.

[5] M. Glover, "Caterpillar's Autonomous Journey - The Argument for Autonomy," SAE Technical Paper 2016-018005, 2016, doi: $10.4271 / 2016-01-8005$.

[6] W. Sun, S. Iwataki, R. Komatsu, H. Fujii, A. Yamashita, and H. Asama, "Simultaneous tele-visualization of construction machine and environment using body mounted cameras," in 2016 IEEE International Conference on Robotics and Biomimetics (ROBIO), Dec 2016, pp. 382-387, doi:10.1109/ROBIO.2016.7866352.

[7] C. A. James, T. P. Bednarz, K. Haustein, L. Alem, C. Caris, and A. Castleden, "Tele-operation of a mobile mining robot using a panoramic display: an exploration of operators sense of presence," in 2011 IEEE International Conference on Automation Science and Engineering, Aug 2011, pp. 279-284, doi:10.1109/CASE.2011.6042427.

[8] C. L. Fernando, M. H. D. Saraiji, Y. Seishu, N. Kuriu, K. Minamizawa, and S. Tachi, "Effectiveness of Spatial Coherent Remote Drive Experience with a Telexistence Backhoe for Construction Sites," presented at the International Conference on Artificial Reality and Telexistence Eurographics Symposium on Virtual Environments, 2015, doi:10.2312/egve.20151312.

[9] J. A. Marshall, P. F. Murphy, and L. K. Daneshmend, "Toward autonomous excavation of fragmented rock: Fullscale experiments," IEEE Transactions on Automation Science and Engineering, vol. 5, no. 3, pp. 562-566, 2008, doi:10.1109/TASE.2007.912709.

[10] A. A. Dobson, J. A. Marshall, and J. Larsson,"Admittance control for robotic loading : Underground field trials with an LHD," in Proc. 10th Conference on Field and Service Robotics, vol. 113, Toronto, Canada, 2016, pp. 487-500, doi:10.1007/978-3-319-27702-8_32.

[11] R. Hill, C. Madden, A. D. Hengel, H. Detmold, and A. Dick, "Measuring latency for video surveillance systems," in Proc. 2009 Digital Image Computing: Techniques and Applications, Dec 2009, pp. 89-95, doi: 10.1109/DICTA.2009.23.

[12] L. H. Zupan, R. J. Peterka, and D. M. Merfeld, "Neural processing of gravito-inertial cues in humans influence of the semicircular canals following post-rotatory tilt," Journal of Neurophysiology, vol. 84, no. 4, 2000, doi: 10.1152/jn.2000.84.4.2001.

[13] S. Dadhich, U. Bodin, F. Sandin, and U. Andersson, "Machine learning approach to automatic bucket loading," in Proc. $201624^{\text {th }}$ IEEE Mediterranean Conference on Control and Automation (MED), 2016, pp. 1260-1265, doi: 10.1109/MED.2016.7535925. 